\title{
Die bronchopulmonale Salmonellose - eine Fallserie und Literaturübersicht
}

\section{Broncho-pulmonary Salmonellosis - A Case Series and Review of the Literature}

Autoren

Institute
M. Jüch' ${ }^{1}$ E. Grabert ${ }^{1}$, W. Rabsch ${ }^{2}$, H. J. Achenbach ${ }^{1}$

${ }^{1}$ Klinik für Pneumologie, Allergologie, Schlaf- und Beatmungsmedizin und thorakale Onkologie, Lungenklinik Lostau gGmbH (Chefarzt Dr. med. H. J. Achenbach)

${ }^{2}$ Nationales Referenzzentrum für Salmonellen und andere bakterielle Enteritiserreger am Robert Koch-Institut (Leitung Prof. Dr. A. Flieger) eingereicht 10.11 .2012 akzeptiert nach Revision 29. 11.2012

Bibliografie

DOI http://dx.doi.org/ 10.1055/s-0032-1326027 Online-Publikation: 17.1.2013 Pneumologie 2013; 67: 174-178 (c) Georg Thieme Verlag KG Stuttgart · New York ISSN 0934-8387

Korrespondenzadresse Dr. med. Mathias Jüch Klinik für Pneumologie, Allergologie, Schlaf- und Beatmungsmedizin und thorakale Onkologie Lungenklinik Lostau gGmbH Lindenstr. 2

39291 Lostau

m.juech@lungenklinik-lostau.de

\section{Zusammenfassung}

\section{$\nabla$}

Bronchopulmonale Salmonellosen sind eine seltene Manifestation extraintestinaler fokaler Salmonellenerkrankungen im Erwachsenenalter. Sie treten auch ohne vorausgegangene gastroenteritische Symptomatik insbesondere bei Patienten über 60 Jahren, abwehrgeschwächten und chronisch lungenkranken Patienten auf. Die Übertragung erfolgt sowohl hämatogen als auch durch Aspiration kontaminierten Mageninhalts. Komplikationen sind das acute respiratory distress syndrome, Lungenabszesse und Pleuraempyeme. Da gehäuft komplikationsreiche und tödliche Verläufe vorkommen, sollte in jedem Fall eine ausreichend lange resistogrammgerechte antibiotische Therapie mit Cephalosporinen der 3.Generation, Trimethoprim/Sulfamethoxazol, Ampicillin oder Fluorchinolonen erfolgen. In dieser Übersichtsarbeit werden 4 unterschiedliche klinische Verläufe bronchopulmonaler Salmonellosen durch Salmonella enterica subsp. enterica Serovar Brandenburg sowie Salmonellen der Gruppen B (04), C (06) und 011 bis 067 dargestellt und im Kontext der aktuellen Literatur diskutiert.

\section{Einleitung}

$\nabla$

Salmonellen sind gramnegative, fakultativ anaerobe, begeißelte Stäbchenbakterien, die abhängig vom vorliegenden Serovar und von Wirtsfaktoren unterschiedliche Krankheitsmanifestationen verursachen können. Die Taxonomie der Salmonellen erfolgt nach dem Kauffmann-White-Le-MinorSchema und orientiert sich an ihren serologisch unterschiedenen Oberflächenantigenen. Die korrekte Bezeichnung für Salmonella Typhi lautet nach der gültigen Nomenklatur Salmonella enterica subspecies enterica Serovar Typhi oder kurz Salmonella Typhi, da S. Typhi nicht mehr als eigene Spezies, sondern als ein Serovar der Spezies

\section{Abstract \\ $\nabla$}

Broncho-pulmonary salmonelloses are rare manifestations of extraintestinal focal infections in adults caused by different subspecies of Salmonella. They may appear without previous gastroenteritic symptoms especially in patients older than 60 years, as well as in patients who are immunocompromised or suffer from chronic pulmonary diseases. The transmission route is hematogenous or via aspiration of contaminated gastric fluid. Complications are acute respiratory distress syndrome, lung abscesses and pleural empyemas. Complicated und lethal courses have been described frequently. Therefore, antibiotic therapy should be initiated in every case with sufficient duration of treatment and in accordance to antibiotic resistance. Recommended antibiotics are third generation cephalosporins, trimethoprim-sulfamethoxazole, ampicillin or fluoroquinolones. In this review 4 different clinical courses of broncho-pulmonary salmonellosis caused by Salmonella enterica subsp. enterica serovar Brandenburg, Salmonella groups B (04), $\mathrm{C}(06)$ and 011 to 067 are presented and discussed in the context of the current literature.

S. enterica betrachtet wird. 2610 Serovare sind gegenwärtig im aktuellen Supplement vom WHO Collaborating Centre for Reference and Research on Salmonella, Institut Pasteur, Paris beschrieben [1]. Um die Übersicht zu erleichtern, wurden Gruppen gebildet, die durch bestimmte O-Antigene charakterisiert sind. Aus klinischer Sicht ist eine Unterscheidung in typhoide und nichttyphoide Salmonellen praktikabel und in der Literatur akzeptiert. Letztere sind eine häufige Ursache der akuten, fieberhaften und selbstlimitierenden Gastroenteritis. Seltener kommt es zur Bakteriämie mit oder ohne extraintestinale fokale Infektion, die in der Regel eine antibiotische Therapie erforderlich macht. S. Typhi sowie S. Paratyphi A, 
B und C verursachen das Vollbild des Typhus bzw. Paratyphus abdominalis mit treppenförmigem Fieberanstieg, teilweise blutiger Diarrhoe, Benommenheit (griechisch typhos=Nebel), Leukopenie, relativer Bradykardie und Roseolen an der Bauchwand. Insbesondere aus Infektionen mit $S$. Typhi kann ein asymptomatisches Dauerausscheidertum resultieren.

Zwischen 2002 und 2012 konnten an der Lungenklinik Lostau in der kulturellen mikrobiologischen Routinediagnostik von mehr als 25.000 Bronchialsekreten in 4 Fällen Salmonellen der Gruppen B (04), C (06) sowie 011 bis 067 nachgewiesen werden. Die unterschiedlichen Verläufe dieser im Folgenden bronchopulmonale Salmonellose genannten extraintestinalen Krankheitsmanifestation werden kurz dargestellt und anschließend im Lichte der aktuellen Literatur diskutiert.

\section{Fall 1}

Bei einer 65-jährigen Patientin mit schwergradiger chronisch obstruktiver Lungenerkrankung (COPD), chronisch hyperkapnischer ventilatorischer Insuffizienz und invasiver Heimbeatmung über ein Tracheostoma werden im Januar 2012 im Rahmen einer Überprüfung der Heimbeatmungstherapie im Bronchialsekret erstmals Salmonellen der Gruppe B (04) nachgewiesen. Die Differenzierung der Salmonelle im Nationalen Referenzzentrum für Salmonellen und andere bakterielle Enteritiserreger am Robert Koch-Institut ergab S. Brandenburg mit der Antigenformel 04:1, v:enz15. Das Antibiogramm wies eine Empfindlichkeit gegen 17 gängige Antibiotika, einschließlich $\beta$-Lactam-Antibiotika, auf. Im Stuhl wurde keine Salmonelle nachgewiesen. Vorausgegangene gastroenteritische Symptome werden von der Patientin verneint. Jedoch war im März 2009 aufgrund einer Pseudoobstruktion des Darms eine Ileozökalresektion erfolgt. Paraklinisch und anamnestisch findet sich kein Hinweis für eine dauerhafte schwere Immunsuppression. Aufgrund häufiger Exazerbationen der COPD wird die Patientin jedoch intermittierend systemisch mit Kortikosteroiden therapiert und erhält eine Dauertherapie mit Protonenpumpeninhibitoren. Bei der Patientin wird eine ambulante resistogrammgerechte Eradikationstherapie mit $2 \times 750 \mathrm{mg}$ Sultamicillin über 14 Tage eingeleitet. Im August 2012 wird sie im Rahmen einer Exazerbation der COPD erneut bronchoskopiert. Weiterhin werden Salmonellen der Gruppe B und Enterobacter cloacae nachgewiesen. Die Stuhldiagnostik bleibt erneut negativ. Nach oraler antibiotischer Therapie mit $2 \times 960 \mathrm{mg}$ Trimethoprim/Sulfamethoxazol über 14 Tage gelingt kein Salmonellennachweis im Bronchialsekret mehr. Die nachträgliche weitere Differenzierung des Erregers ergab erneut $S$. Brandenburg. Eine Reduktion der Exazerbationsfrequenz nach Abschluss der Eradikationstherapie konnte nicht nachgewiesen werden.

\section{Fall 2}

$\nabla$

Bei einem 66-jährigen Patienten mit kurativ behandeltem distalen Ösophaguskarzinom mit Zustand nach Ösophagusteilresektion und Ösophagogastrostomie und stabiler koronarer Herzerkrankung wird im Januar 2009 ein Plattenepithelkarzinom der Lunge im Stadium T2NxM0 diagnostiziert. Im Rahmen der Narkoseeinleitung für die geplante Mediastinoskopie kommt es zum Aspirationsgeschehen. In der sofort durchgeführten Bronchoskopie wird Bronchialsekret zur mikrobiologischen Untersuchung gewonnen. Es gelingt der Nachweis von Salmonellen der Gruppe
C (06), die leider nicht weiter differenziert wurden. Im Resistogramm ist die Salmonelle sensibel gegenüber 15 gängigen Antibiotika mit Ausnahme von Doxycyclin. Eine mikrobiologische Stuhldiagnostik auf Salmonellen bleibt negativ. Der Patient erhält eine kombinierte intravenöse antibiotische Therapie mit $1 \times 2 \mathrm{~g}$ Ceftriaxon, $3 \times 600 \mathrm{mg}$ Clindamycin und $3 \times 500 \mathrm{mg}$ Metronidazol und wird invasiv beatmet. Es entwickelt sich das Vollbild einer pneumogenen Sepsis mit massivem Anstieg der Entzündungsparameter, katecholaminpflichtiger hämodynamischer Instabilität und akutem Nierenversagen. Röntgenologisch zeigen sich progrediente fleckig konfluierende Infiltrate im rechten Lungenunterlappen. Ein Keimnachweis in der Blutkultur gelingt nicht. Der Patient verstirbt 4 Tage nach dem Aspirationsereignis.

\section{Fall 3}

Bei einem 51-jährigen Patienten mit Zustand nach Pneumonektomie links bei Plattenepithelkarzinom der Lunge im UICC-Stadium IIA werden im Dezember 2006 im Rahmen der Nachsorge 6 Monate postoperativ im Bronchialsekret Salmonellen der Gruppe 011 bis 067 nachgewiesen. Die Salmonelle ist sensibel gegenüber allen 15 getesteten Antibiotika. Der Patient ist klinisch asymptomatisch. Von einer antibiotischen Therapie wird daher abgesehen. In einer erneuten bronchoskopischen Kontrolle 5 Monate später gelingt kein Keimnachweis mehr.

\section{Fall 4}

Bei einem 67-jährigen Patienten wird im August 2004 ein kleinzelliges Lungenkarzinom im UICC-Stadium IA im rechten Unterlappen diagnostiziert. Einige Tage nach der kurativ intendierten Lobektomie entwickelt der Patient heftige wässrige Diarrhöen, Fieber und einen erheblichen Anstieg des C-reaktiven Proteins (CrP) bei normwertigen Leukozytenzahlen. Sowohl in den Stuhlproben als auch in der Blutkultur gelingt der Nachweis von Salmonellen der Gruppe B (04). Eine weitere Differenzierung erfolgt nicht. Im Resistogramm werden 15 gängige Antibiotika getestet. Es werden Resistenzen gegenüber Aminopenicillinen und Doxycyclin nachgewiesen. Da keine Resistenz gegen Fluorchinolone besteht, erhält der Patient eine intravenöse antibiotische Therapie mit $2 \times 400 \mathrm{mg}$ Ciprofloxacin. Dennoch kommt es zu einer Verschlechterung der respiratorischen Situation mit Dyspnoe und hypoxischer respiratorischer Insuffizienz, die eine kontinuierliche nasale Sauerstoffgabe erforderlich macht. Röntgenologisch zeigen sich eine milchglasartige Eintrübung und kleinfleckig konfluierende Infiltrate im rechten Oberlappen ( $\bullet$ Abb.1). CT-morphologisch bestehen ausgedehnte Infiltrationen der rechten Lunge mit Konsolidierungen, Air-Bronchogramm, angedeuteten Honigwaben und Bronchusdilatationen ( Abb.2). Auch im Bronchialsekret gelingt der Nachweis von Salmonellen der Gruppe B. Unter Fortsetzung der antibiotischen Therapie entfiebert der Patient, das $\mathrm{CrP}$ ist rückläufig, die respiratorische Situation bessert sich. Von einer zusätzlichen hochdosierten Dexamethasongabe wird daher abgesehen. In der mikrobiologischen Diagnostik aus Stuhlproben und Bronchialsekret 10 Tage später können keine Salmonellen mehr nachgewiesen werden. Die antibiotische Therapie wird nach 14 Tagen beendet. Röntgen- und CTmorphologisch zeigt sich in den folgenden Monaten eine langsame Regredienz der pulmonalen Befunde. In einer erneuten Stuhlprobe 13 Tage nach Beginn der antibiotischen Therapie wer- 


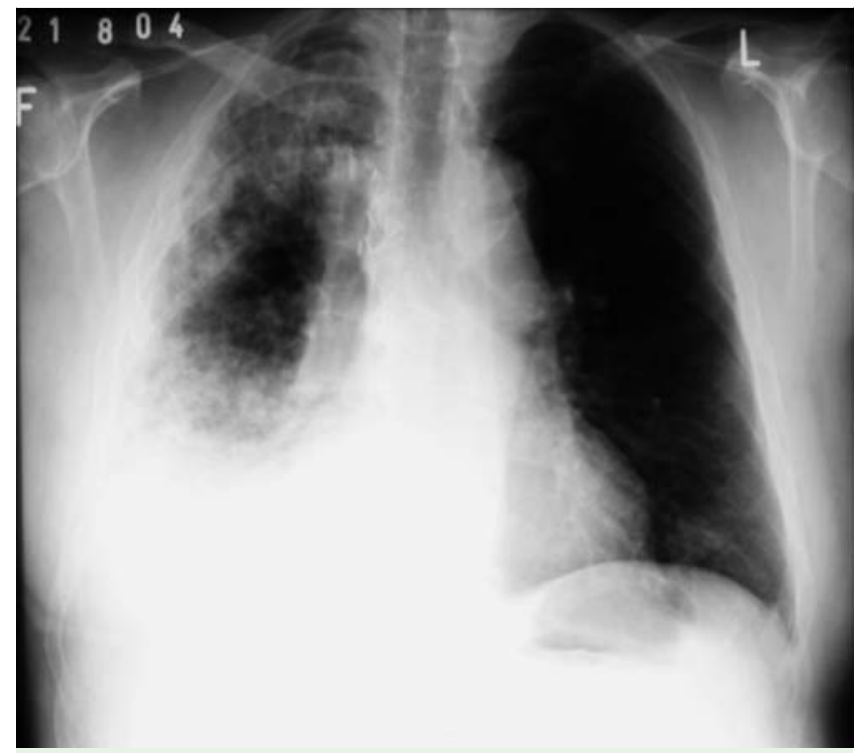

Abb.1 Röntgen-Thorax-Aufnahme in p.a. Projektion des Patienten aus Fall 4. Milchglasartige Eintrübung und kleinfleckig konfluierende Infiltrate im rechten Oberlappen. Pleuraerguss rechts. Zustand nach Unterlappenresektion rechts.

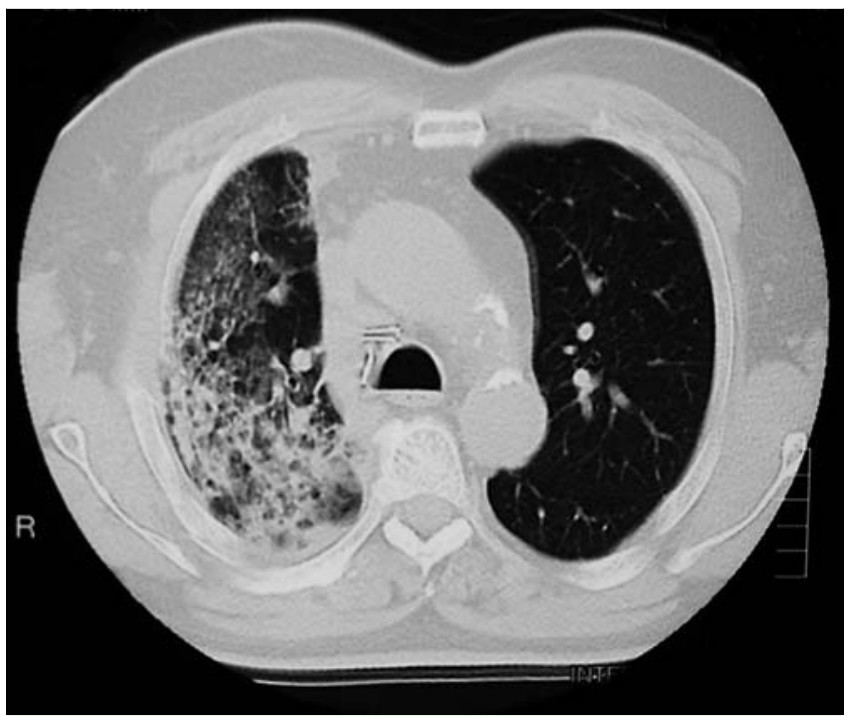

Abb. 2 CT-Thorax des Patienten aus Fall 4 (Transversalschnitt, Lungenfenster, ohne Kontrastmittel). Ausgedehnte Infiltrationen der rechten Lunge mit Konsolidierungen, Air-Bronchogramm und Bronchusdilatationen.

den keine Salmonellen mehr nachgewiesen. Eine Wiederholung der Stuhldiagnostik in der ambulanten Weiterbetreuung erfolgt nicht.

\section{Diskussion}

$\nabla$

Extraintestinale Manifestationen nichttyphöser Salmonellosen im Erwachsenenalter kommen immer wieder vor [2]. Sie sind mit einem schwereren Krankheitsverlauf, häufigerer und längerer Hospitalisation und höheren Kosten für das Gesundheitssystem vergesellschaftet als die enteritische Verlaufsform $[3,4]$. In einer Untersuchung einer spanischen Arbeitsgruppe an einem Krankenhaus der Grundversorgung zeigten $8 \%$ aller Salmonellosen einen extraintestinalen Manifestationsort [5]. Eine vorausge-
Tab. 1 Extraintestinale fokale Infektionen durch Salmonellen.

Intraabdominell:
Leber-, Milz-, Tuboovarialabszesse, Gallenblasenempyem, Peritonitis,
Pyelonephritis
Respirationstrakt:
Tracheobronchitis, Pneumonie, Lungenabszess, Pleuraempyem
Osteoartikulär:
Osteomyelitis, Arthritis
Kardiovaskulär:
Mykotisches Aneurysma, Endokarditis, Perikarditis
Zentralnervös:
Meningitis, Hirnabszess
Sonstige:
Haut- und Weichteilabszesse

Tab. 2 Risikofaktoren für das Auftreten extraintestinaler fokaler Salmonelleninfektionen.

\begin{tabular}{l}
\hline Allgemeine Risikofaktoren für extraintestinale fokale Salmonellosen: \\
\hline Lebensalter> 50 Jahre \\
\hline Autoimmunerkrankungen \\
\hline Medikamentöse Immunsuppression, einschließlich Chemotherapie \\
\hline HIV-Infektion \\
\hline Antikörpermangelsyndrome \\
\hline Alkoholismus \\
\hline Solide und hämatologische Neoplasien \\
\hline Dauertherapie mit Antazida \\
\hline Spezielle zusätzliche Risikofaktoren für bronchopulmonale \\
Salmonellosen: \\
Lebensalter>60 Jahre \\
\hline Chronische Lungenerkrankungen (COPD, Bronchiektasen, Lungen- \\
karzinom) \\
\hline Ösophagotracheale und -bronchiale Fisteln \\
\hline Schwere Thoraxwanddeformitäten \\
\hline
\end{tabular}

gangene gastroenteritische Symptomatik war dabei nicht immer vorhanden. In Übereinstimmung mit bereits bekannten Daten gelang der Salmonellennachweis am häufigsten im Blut ohne weiteren infektiösen Fokus, gefolgt von fokalen Infektionen in den ableitenden Harnwegen, dem unteren Respirationstrakt, intraabdominell sowie an vaskulären, osteoartikulären und zentralnervösen Manifestationsorten [6-9] ( Tab. 1). Zudem wurden Endokarditiden und Weichteilabszesse beschrieben $[8,9]$. Extraintestinale Salmonellosen treten häufiger bei den Serovaren S. Paratyphi A, B und C, S. Dublin und S. Choleraesuis der Subspezies enterica auf; zunehmend werden auch Salmonellen der Subspezies II bis IV nachgewiesen [10]. Ein erhöhtes Risiko für das Auftreten extraintestinaler fokaler Salmonellosen im Allgemeinen besteht zudem bei einer Reihe von Wirtsfaktoren ( $\bullet$ Tab.2). Hierzu zählen ein Lebensalter über 50 Jahre [5,10,11], Autoimmunerkrankungen wie systemischer Lupus erythematodes [12] oder die granulomatöse Polyangiitis [13], die eine immunsuppressive Therapie, einschließlich einer systemischen Dauertherapie mit Kortikosteroiden, erfordern, eine chemotherapeutische Behandlung, insbesondere mit Substanzen, die ein hohes Mucositis-Risiko bergen [14,15], eine HIV-Infektion [16,17], ein Antikörpermangelsyndrom [18], Alkoholismus [19,20], hämatologische Neoplasien und solide Malignome [21,22] sowie eine Dauertherapie mit Antazida [20,23]. Zudem besteht ein erhöhtes Risiko für extraintestinale fokale Salmonellosen durch die Subspezies II bis IV bei durch Reptilien und Amphibien übertragenen Salmonellosen [24]. Für bronchopulmonale Salmonellosen im 
Speziellen prädisponieren ein Lebensalter über 60 Jahre [11], chronische Lungenerkrankungen, einschließlich des Lungenkarzinoms [7,25], ösophagotracheale oder -bronchiale Fisteln sowie schwere Thoraxwanddeformitäten [26]. Salmonellen der Subspezies I, IIIa und IIIb scheinen häufiger bronchopulmonale Salmonellosen zu verursachen $[5,10]$. Dabei können Salmonellen sowohl hämatogen als auch durch Aspiration von kontaminiertem Mageninhalt in das Tracheobronchialsystem gelangen. Während in der 4. Fallvorstellung dieser Übersichtsarbeit ein hämatogener Infektionsweg bei Nachweis von Salmonellen im Stuhl, in der Blutkultur und im Bronchialsekret als bewiesen angesehen werden kann, kann im 2. Fall eine Übertragung durch Aspiration kontaminierten Mageninhalts bei Zustand nach Resektion eines distalen Ösophaguskarzinoms und Magenhochzug vermutet werden. Genau wie im 1.Fall bestand auch hier eine Dauertherapie mit Protonenpumpeninhibitoren. Im 1. Fall lag zudem eine intermittierende immunsuppressive Therapie mit systemischen Kortikosteroiden vor. Bei der Tracheostomaträgerin muss zudem eine Schmierinfektion während der selbstständig durchgeführten Tracheostomapflege als möglicher Infektionsweg erwogen werden. Bis auf den Patienten im 4. Fall ließ sich bei den hier präsentierten Fällen in Übereinstimmung mit den Daten anderer Arbeitsgruppen [5] keine vorausgegangene gastroenteritische Symptomatik eruieren. Für eine Lungenklinik wenig überraschend lag bei allen 4 Patienten eine chronische Lungenerkrankung vor. In 2 Fällen lagen Lungenkarzinome in niedrigen Tumorstadien ohne vorausgegangene systemische tumorspezifische Therapie vor. Im 3. Fall handelte es sich um einen Zustand nach Pneumonektomie bei kurativ operiertem Lungenkarzinom. Die Patientin im 1. Fall hatte eine schwergradige COPD. Alle hier präsentierten Patienten waren über 50 Jahre alt. 3 der 4 Patienten gehörten zu der auch in wesentlich größeren Patientenkollektiven nachgewiesenen Risikogruppe mit einem Lebensalter über 60 Jahren [11].

Bronchopulmonale Salmonellosen sind mit einer hohen Mortalität von teilweise mehr als $60 \%$ vergesellschaftet [27]. Komplikationen sind der Lungenabszess, das Pleuraempyem [7,28] sowie das acute respiratory distress syndrome [25]. Auch im 2. hier präsentierten Fall entwickelte sich im Rahmen einer pneumogenen Sepsis ein fulminanter und letaler Krankheitsverlauf. Jedoch sind dabei eine zusätzliche chemische Schädigung der Alveolen durch sauren Magensaft und eine vorbestehende stabile koronare Herzerkrankung als relevante Kofaktoren zu berücksichtigen. Während in den vorgestellten Fällen die genannten Akutkomplikationen nicht auftraten, konnten wir ein bronchopulmonales Langzeitausscheidertum über 8 Monate nachweisen, das möglicherweise eine Exazerbation der vorbestehenden COPD getriggert hat. In Anlehnung an das Dauerausscheidertum im biliodigestiven und harnableitenden System sollte der Begriff des bronchopulmonalen Dauerausscheidertums erst ab einem asymptomatischen Keimnachweis im Bronchialsekret über mehr als 12 Monate verwendet werden. Ein solches bronchopulmonales Dauerausscheidertum über 18 Monate wurde bislang in der Literatur erst bei einem einzigen Patienten mit endobronchialem Aspergillom nach einem rektalchirurgischen Eingriff beschrieben [29]. Interessanterweise hatte sich die Patientin im 1. Fall ebenfalls einem länger zurückliegenden kolorektalchirurgischen Eingriff unterzogen.

Die mikrobiologische Identifizierung der Erreger bei bronchopulmonaler Salmonellose erfolgt aus der Bronchialspülung oder der bronchoalveolären Lavage und nimmt zwischen 2 und 3 Tage in Anspruch. Nach Kultivierung auf Mac-Conkey-Agar oder selekti- ver auf Hektoen-Agar erfolgt die weitere biochemische und serologische Differenzierung. Die Bestimmung der Serovare und eine anschließende Feindifferenzierung auch mit molekularen Methoden ist am Nationalen Referenzzentrum für Salmonellen und andere bakterielle Enteritiserreger am Robert Koch-Institut in Wernigerode möglich und sollte besonders auch bei extraintestinalen Nachweisen genutzt werden. In den vorliegenden Fällen ist eine Feindifferenzierung lediglich in einem Fall erfolgt. In den übrigen 3 Fällen wurde mit kommerziell erhältlichen AntikörperKits eine Gruppendifferenzierung anhand der Oberflächenantigene in die Gruppen 04 (Gruppe B), 06 (Gruppe C) sowie 011 bis 067 und eine Resistenztestung durchgeführt, was für die klinische Fragestellung durchaus ausreichend sein kann [30]. Der Nachweis von Salmonellen im Bronchialsekret ist wie jede andere Salmonellose laut Infektionsschutzgesetz meldepflichtig, soweit der Nachweis auf eine akute Infektion hinweist.

Zur antibiotischen Therapie der bronchopulmonalen Salmonellosen können Cephalosporine der 3. Generation, Trimethoprim/ Sulfamethoxazol, Ampicillin oder Fluorchinolone eingesetzt werden [30]. Die Therapie sollte resistogrammgerecht erfolgen, da Resistenzen auch in Europa zunehmend Probleme bereiten [31]. Die Therapiedauer sollte zwischen 10 und 14 Tage betragen [30] und der Therapieerfolg nach Abschluss der Behandlung durch erneute mikrobiologische Diagnostik aus dem Bronchialsekret überprüft werden. Bei schwerer invasiver Salmonellose kann zudem eine Dexamethason-Therapie ( $3 \mathrm{mg} / \mathrm{kg}$ Körpergewicht loading dose, nachfolgend $1 \mathrm{mg} / \mathrm{kg}$ Körpergewicht) erwogen werden [32].

\section{Schlussfolgerung \\ $\nabla$}

Bronchopulmonale Salmonellosen sind eine seltene Manifestation extraintestinaler fokaler Salmonellenerkrankungen im Erwachsenenalter. Sie treten auch ohne vorausgegangene gastroenteritische Symptomatik insbesondere bei älteren, abwehrgeschwächten und chronisch lungenkranken Patienten auf. Da gehäuft komplikationsreiche und tödliche Verläufe vorkommen, sollte in jedem Fall eine ausreichend lange resistogrammgerechte antibiotische Therapie erfolgen. Eine serologische Typisierung im Nationalen Referenzzentrum für Salmonellen und andere bakterielle Enteritis-Erreger sollte angestrebt werden.

\section{Danksagung \\ $\nabla$}

Die Autoren bedanken sich bei Frau Dr. med. Karin Ludwig, Chefärztin der Klinik für Radiologie der Lungenklinik Lostau, für die Bereitstellung der Röntgen- und CT-Aufnahmen sowie bei Frau Dr. Fruth und Marita Wahnfried am Nationalen Referenzzentrum für Salmonellen und andere bakterielle Enteritis-Erreger für die Typisierung.

\section{Widmung \\ $\nabla$}

Die Autoren widmen diesen Artikel Herrn Prof. Dr. med. habil. Heinrich Friedel (2.5.1920 - 21.6.2012), dem langjährigen ärztlichen Direktor und Chefarzt der Lungenklinik Lostau. 


\section{Interessenkonflikt}

Die Autoren geben an, dass kein Interessenkonflikt besteht.

\section{Literatur}

1 Guibourdenche M, Roggentin P, Mikoleit M et al. Supplement 20032007 (No. 47) to the White-Kauffmann-Le Minor scheme. Res Microbiol 2010; 161: $26-29$

2 Weiss SH, Blaser MJ, Paleologo FP et al. Occurrence and distribution of serotypes of the Arizona subgroup of Salmonella strains in the United States from 1967 to 1976. J Clin Microbiol 1986; 23: 1056-1064

3 Chen PL, Li CY, Hsieh TH et al. Epidemiology, disease spectrum and economic burden of non-typhoidal Salmonella infections in Taiwan, 2006-2008. Epidemiol Infect 2012: 1-8

4 Trevejo RT, Courtney JG, Starr M et al. Epidemiology of salmonellosis in California, 1990-1999: morbidity, mortality, and hospitalization costs. Am J Epidemiol 2003; 157: 48-57

5 Rodriguez M, de Diego I, Mendoza MC. Extraintestinal salmonellosis in a general hospital (1991 to 1996): relationships between Salmonella genomic groups and clinical presentations. J Clin Microbiol 1998; 36 : $3291-3296$

6 Rodriguez M, de Diego I, Martinez $N$ et al. Nontyphoidal Salmonella causing focal infections in patients admitted at a Spanish general hospital during an 11-year period (1991-2001). Int J Med Microbiol 2006; 296: $211-222$

7 Cohen JI, Bartlett JA, Corey GR. Extra-intestinal manifestations of salmonella infections. Medicine (Baltimore) 1987; 66: 349-388

8 Huang DB, DuPont HL. Problem pathogens: extra-intestinal complications of Salmonella enterica serotype Typhi infection. Lancet Infect Dis 2005; 5: $341-348$

9 Wilkins EG, Roberts C. Extraintestinal salmonellosis. Epidemiol Infect 1988; 100: $361-368$

10 Abbott SL, Ni FC, Janda JM. Increase in extraintestinal infections caused by Salmonella enterica subspecies II-IV. Emerg Infect Dis 2012; 18: 637-639

11 Chen PL, Lee HC, Lee NY et al. Non-typhoidal Salmonella bacteraemia in elderly patients: an increased risk for endovascular infections, osteomyelitis and mortality. Epidemiol Infect 2012; 140: 2037-2044

12 Gerona JG, Navarra SV. Salmonella infections in patients with systemic lupus erythematosus: a case series. Int J Rheum Dis 2009; 12: 319323

13 Chan JC, Raffin TA. Salmonella lung abscess complicating Wegener's granulomatosis. Respir Med 1991; 85: 339-341

14 Delaloye J, Merlani G, Petignat C et al. Nosocomial nontyphoidal salmonellosis after antineoplastic chemotherapy: reactivation of asymptomatic colonization? Eur J Clin Microbiol Infect Dis 2004; 23: 751 - 758
15 Souglakos J, Kotsakis A, Kouroussis C et al. Nonneutropenic febrile episodes associated with docetaxel-based chemotherapy in patients with solid tumors. Cancer 2002; 95: 1326-1333

16 Sperber SJ, Schleupner CJ. Salmonellosis during infection with human immunodeficiency virus. Rev Infect Dis 1987; 9: 925-934

17 Casado JL, Navas E, Frutos B et al. Salmonella lung involvement in patients with HIV infection. Chest 1997; 112: 1197-1201

18 Mamishi S, Eghbali AN, Rezaei $N$ et al. A single center 14 years study of infectious complications leading to hospitalization of patients with primary antibody deficiencies. Braz J Infect Dis 2010; 14: 351 - 355

19 Segado A, Quero F, Vidal JL et al. [Necrotizing pneumonia by Salmonella in an alcoholic patient]. An Med Interna 2003; 20: 108-109

20 Genzen JR, Towle DM, Kravetz JD et al. Salmonella typhimurium pulmonary infection in an immunocompetent patient. Conn Med 2008; 72 : $139-142$

21 Wolfe MS, Louria DB, Armstrong D et al. Salmonellosis in patients with neoplastic disease. A review of 100 episodes at Memorial Cancer Center over a 13-year period. Arch Intern Med 1971; 128: 546-554

22 Han T, Sokal JE, Neter E. Salmonellosis in disseminated malignant diseases. A seven-year review (1959-1965). N Engl J Med 1967; 276: $1045-1052$

23 Schapira M, Roquet ME, Henrion J et al. Severe nontyphoidal salmonellosis probably in relation with omeprazole treatment: report of 2 cases. Acta Gastroenterol Belg 1996; 59: 168-169

24 Centers for Disease Control and Prevention. Reptile-associated salmonellosis-selected states, 1998-2002. MMWR Morb Mortal Wkly Rep 2003; 52: 1206 - 1209

25 Samonis G, Maraki S, Kouroussis C et al. Salmonella enterica pneumonia in a patient with lung cancer. J Clin Microbiol 2003; 41: 5820-5822

26 Yossepowitch O, Sviri S, Ben-Yehuda A et al. Salmonella infection and pneumonia in a patient with kyphoscoliosis. Eur J Med Res 1996; 1: $589-590$

27 Aguado JM, Obeso G, Cabanillas JJ et al. Pleuropulmonary infections due to nontyphoid strains of Salmonella. Arch Intern Med 1990; 150: 54 56

28 Satue JA, Aguado JM, Ramon Costa J et al. Pulmonary abscess due to non-typhi Salmonella in a patient with AIDS. Clin Infect Dis 1994; 19: $555-557$

29 Reiss-Levy E, Isaacs F, Tully $M$ et al. Chronic pulmonary infection with Salmonella typhimurium. Med J Aust 1980; 2: 674-675

30 Hohmann EL. Nontyphoidal salmonellosis. Clin Infect Dis 2001; 32: $263-269$

31 Casin I, Breuil J, Brisabois A et al. Multidrug-resistant human and animal Salmonella typhimurium isolates in France belong predominantly to a DT104 clone with the chromosome- and integron-encoded betalactamase PSE-1. J Infect Dis 1999; 179: 1173-1182

32 Hoffman SL, Punjabi NH, Kumala S et al. Reduction of mortality in chloramphenicol-treated severe typhoid fever by high-dose dexamethasone. N Engl J Med 1984; 310: 82 - 88 